\title{
Carbohydrates to Prevent and Treat Obesity in a Murine Model of Diet- Induced Obesity
}

\author{
Ellen Vercalsteren ${ }^{\mathrm{a}}$ Christine Vranckx $^{\mathrm{a}}$ Katrien Corbeels ${ }^{\mathrm{b}}$ \\ Bart Van der Schueren ${ }^{b, c}$ Greetje Vande Velde ${ }^{d}$ Roger Lijnen ${ }^{a}$ Ilse Scroyen ${ }^{\text {a, e }}$ \\ ${ }^{a}$ Department of Cardiovascular Sciences, KU Leuven, Leuven, Belgium; bepartment of Chronic Diseases, \\ Metabolism and Ageing, KU Leuven, Leuven, Belgium; 'Department of Endocrinology, University Hospitals Leuven, \\ Leuven, Belgium; dDepartment of Imaging and Pathology, KU Leuven, Leuven, Belgium; eHealth Department, \\ University Colleges Leuven-Limburg, Leuven, Belgium
}

\section{Keywords \\ Adipose tissue · Inflammation · Animal models · Obesity · Carbohydrates}

\begin{abstract}
Introduction: The biggest risk factor for obesity and its associated comorbidities is a Western diet. This Western diet induces adipose tissue (AT) inflammation, which causes an AT dysfunction. Since AT is a vital endocrine organ, its dysfunction damages other organs, thus inducing a state of chronic inflammation and causing various comorbidities. Even though it is evident a Western diet, high in fat and carbohydrates, induces obesity and its complications, it is not known yet which macronutrient plays the most important role. Therefore, the aim of this study was to investigate the effect of macronutrient composition on obesity and to reverse the Western diet-induced metabolic risk via caloric restriction (CR) or a change of diet composition. Materials and Methods: Male, C57BL/6JRj mice were fed with a diet high in fat, sucrose, fructose, sucrose and fructose, starch, a Western diet, or a control diet for 15 weeks. To assess reversibility of the metabolic risk, mice were first made obese via 15 weeks of WD and then put on either a CR or switched to a sucrose-
\end{abstract}

rich diet. Results: A sucrose-rich and high-starch diet induced less obesity and a better metabolic profile than a Western diet, evidenced by less hepatic steatosis, lower plasma cholesterol, and less insulin resistance. Furthermore, these diets induced less intra-abdominal AT inflammation than a Western diet, since mRNA levels of pro-inflammatory markers were lower and there was less macrophage infiltration. Expression of tight junction markers in colon tissue was higher in the sucrose-rich and high-starch group than the Western group, indicating a better intestinal integrity upon sucrose-rich and high-starch feeding. Additionally, CR induced weight loss and decreased both metabolic abnormalities and AT inflammation, regardless of macronutrient composition. However, effects were more pronounced upon $C R$ with sucrose-rich or high-starch diet. Even without $C R$, switching obese mice to a sucrose-rich diet induced weight loss and decreased AT inflammation and metabolic aberrations. Discussion: A diet high in sucrose or starch induces less obesity and obesity-associated complications. Moreover, switching obese mice to a sucrose-rich diet elicits weight loss and decreases obesity-induced metabolic complications, highlighting the potential of carbohydrates to treat obesity.

(c) 2021 The Author(s).

Published by S. Karger AG, Basel
C 2021 The Author(s).

Published by S. Karger AG, Basel

This is an Open Access article licensed under the Creative Commons Attribution-NonCommercial-4.0 International License (CC BY-NC) (http://www.karger.com/Services/OpenAccessLicense), applicable to the online version of the article only. Usage and distribution for commercial purposes requires written permission.
Correspondence to:

Ilse Scroyen, ilse.scroyen @ kuleuven.be 


\section{Introduction}

With 650 million adults suffering from obesity globally and its association with many severe comorbidities, it is one of the biggest health challenges of the 21st century [1]. One of the major risk factors for obesity development is a Western diet (WD), high in carbohydrates and fat [2]. This WD causes intra-abdominal adipose tissue (AT) inflammation. Since the AT is a vital endocrine organ, its dysfunction due to the inflammatory state affects numerous other organs, ultimately inducing a state of chronic, systemic, and low-grade inflammation and increasing the risk for metabolic complications [3]. However, despite the clear importance of evidence-based dietary guidelines, the composition of these remains a hotly debated topic [4-6].

Studies conducted in both animal models and humans have shown a positive association between sucrose intake and obesity $[7,8]$. This led to the guidelines issued by the WHO that sugar intake should be limited to $10 \%$ of the daily energy intake [9]. However, in many other studies, both murine and human, dietary sucrose did not induce an obese state $[6,10,11]$. Moreover, Hu et al. [12] demonstrated that high dietary carbohydrate intake does not cause adiposity. These results indicate that there is still no conclusive, scientific evidence for an ideal diet composition.

Accumulating evidence suggests that the gastrointestinal tract plays an important role in low-grade chronic inflammation $[13,14]$. The gut microbiota normally preserves intestinal integrity. A WD causes a dysbiosis that reduces mucosal barrier function, resulting in higher plasma LPS levels that aggravate the chronic inflammation associated with obesity $[15,16]$. Some studies have highlighted a potential beneficial effect of carbohydrates on the gut microbiota [17-20]. Additionally, switching from a WD to a high-carbohydrate diet ameliorates gut microbiota composition and decreases diet-induced inflammation [18]. However, no distinction has been made between different carbohydrate types. Therefore, the aims of this study were (i) to investigate the effects of saturated fat and carbohydrates on the chronic inflammatory state associated with diet-induced obesity in mice and (ii) to investigate different interventions to reduce the WD-induced metabolic risk in mice.

\section{Materials and Methods}

Animal Models

Male, 10-week-old, C57BL/6JRj mice (Janvier Labs, Le Genest Saint Isle, France) were put on a WD, high-fat diet (HFD), sucroserich diet (HSUD), high-fructose diet (HFRD), high-fructose-su- crose diet (HCFD), high-starch diet (HSTD), control diet (SFD), or maintenance diet (MD) for 15 weeks ( $n=10$ per group). A detailed diet composition of these diets (custom-ordered at Sniff Spezialdiäten, Soest, Germany) can be found in Table 1. Animals were housed on average 2-3 per cage, and body weight and food intake were monitored weekly. Obese mice (exposed to WD for 15 weeks) were subjected to (i) a caloric restriction of $30 \%$ on WD, HSUD, or HSTD ( $n=10$ per group) or (ii) a switch to an $\operatorname{HSUD}(n=5)$. Mice subjected to caloric restriction were housed on average 2 per cage and monitored daily. Control mice were continued on WD ad libitum $(n=10)$. After the dietary period, mice were fasted for $6 \mathrm{~h}$, blood glucose was measured, and the animals were anesthetized with an intraperitoneal injection of $60 \mathrm{mg} / \mathrm{kg}$ sodium pentobarbital (Dolethal; Abbott Laboratories, North Chicago, IL, USA). Blood was collected via the retro-orbital sinus on trisodium citrate (final concentration of $0.01 \mathrm{M}$ ), and plasma was stored at $-80^{\circ} \mathrm{C}$. Intra-abdominal (gonadal [GN] and mesenteric [MES]) and subcutaneous (SC) AT, liver, and colon were weighed and stored at $-80^{\circ} \mathrm{C}$ or fixed in $4 \%$ formaldehyde for subsequent analysis.

\section{In vivo Experiments}

\section{Indirect Calorimetry}

Mice were individually housed in automated Phenomaster Calocages (TSE systems, Bad Homburg, Germany) after 14 weeks of diet exposure. After a 7-day adaptation, ambulatory activity, heat production, $\mathrm{O}_{2}$ consumption $\left(\mathrm{VO}_{2}\right)$, and $\mathrm{CO}_{2}$ generation $\left(\mathrm{VCO}_{2}\right)$ data were collected for $72 \mathrm{~h}$ at $22^{\circ} \mathrm{C}$ on a 12 -h day/night cycle. The first $24 \mathrm{~h}$ were excluded from the analysis to avoid environmental cage bias. The energy expenditure was calculated as reported previously [21]. Ambulatory activity was defined as total counts of infrared light beam breaks along the $X$-axis. Results were corrected for body weight, to exclude effects due to weight differences.

\section{Glucose Tolerance Test}

After 14 weeks of diet, mice were fasted for $6 \mathrm{~h}$. Then, blood glucose levels were measured via tail nick, mice were weighed and injected intraperitoneally with $2 \mathrm{mg} / \mathrm{g}$ of a $30 \%$ glucose solution, and then blood glucose levels were measured at 30,60, 90, and 120 $\min$.

\section{Micro-Computed Tomography}

Mice were anesthetized with $2 \%$ isoflurane in $100 \% \mathrm{O}_{2}$ and placed in a small animal CT scanner (Skyscan 1278 software version 4.2; Bruker microCT, Kontich, Belgium). Scanning time was $7 \mathrm{~min}$ with the following parameters: $50-\mathrm{kVp} \mathrm{X}$-ray source, 0.5 aluminum filter, $920-\mu \mathrm{A}$ current, 35-ms exposure time/projection, 4 projections/position with $0.5^{\circ}$ increments, and a total angle of $220^{\circ}$. Data reconstruction and analysis was performed using manufacturer's software.

\section{Metabolic Parameters}

Plasma cholesterol was quantitated using routine clinical assays. Commercially available kits were used to determine plasma insulin (Mercodia, Upsala, Sweden), adiponectin (R\&D systems, Minneapolis, MN, USA), and LPS levels (Thermo Fisher, Waltham, MA, USA) according to the manufacturer's protocol. The Homeostatic Model Assessment of Insulin Resistance (HOMA-IR) index was calculated as follows: fasting glucose levels $(\mathrm{mM}) \times$ fasting insulin levels $(\mathrm{mU} / \mathrm{L}) / 22.5$. 
Table 1. Composition of the diets

\begin{tabular}{|c|c|c|c|c|c|c|c|c|}
\hline & SFD & $\mathrm{MD}$ & WD & HSUD & HFD & HFRD & HCFD & HSTD \\
\hline Energy, MJ/kg & 17.2 & 16.3 & 19.1 & 15.6 & 25.2 & 15.6 & 15.6 & 15.4 \\
\hline Total protein, $\%$ & 40 & 15 & 15 & 15 & 15 & 15 & 15 & 15 \\
\hline Total fat, \% & 30 & 17.5 & 43 & 10 & 75 & 10 & 10 & 10 \\
\hline Total carbohydrates, \% & 30 & 67.5 & 42 & 75 & 10 & 75 & 75 & 75 \\
\hline \multicolumn{9}{|l|}{ Detailed composition, $\%$} \\
\hline Casein & 46.850 & 19.500 & 19.500 & 15.770 & 25.560 & 15.770 & 15.770 & 15.770 \\
\hline Fructose & - & - & - & - & - & 47.842 & 23.912 & - \\
\hline Corn starch, pre-gelatinized & 9.000 & 19.990 & 15.000 & 21.250 & 4.244 & 21.250 & 21.250 & 69.074 \\
\hline Cellulose powder & 5.000 & 5.000 & 5.000 & 5.000 & 5.000 & 5.000 & 5.000 & 5.000 \\
\hline DL-methionine & 0.300 & 0.300 & 0.300 & 0.300 & 0.300 & 0.300 & 0.300 & 0.300 \\
\hline Vitamins & 1.100 & 1.100 & 1.100 & 1.100 & 1.100 & 1.100 & 1.100 & 1.100 \\
\hline Minerals and trace elements & 4.300 & 4.300 & 4.300 & 4.300 & 4.300 & 4.300 & 4.300 & 4.300 \\
\hline Safflower oil & 0.800 & 0.800 & 0.800 & 0.800 & 0.800 & 0.800 & 0.800 & 0.800 \\
\hline Linseed oil & 0.200 & 0.200 & 0.200 & 0.200 & 0.200 & 0.200 & 0.200 & 0.200 \\
\hline
\end{tabular}

SFD, standard-fat diet; MD, maintenance diet; WD, Western diet; HSUD, sucrose-rich diet; HFD, high-fat diet; HFRD, high-fructose diet; HCFD, high-fructose-sucrose diet, HSTD, high-starch diet.

Quantitative Real-Time Polymerase Chain Reaction

RNA extractions were performed using the RNeasy mini kit (Qiagen, Basel, Switzerland) according to manufacturer's instructions. The Multiscribe ${ }^{\mathrm{TM}}$ Reverse Transcriptase kit (Thermo Fisher) was used to transcribe $10 \mathrm{ng} / \mu \mathrm{L}$ RNA into cDNA according to the manufacturer's protocol. Taqman gene expression assays (Thermo Fisher) were used to analyze mRNA levels of F4/80 (Mm00802529_m1), monochemoattractant protein-1 (MCP-1: Mm00441242_m1), and serum amyloid A3 (Mm00441203_m1) in AT and occludin (Occln; Mm00500912_m1), zona occludens-1 (ZO-1; Mm00493699_m1), and Saa3 in the colon by RT-qPCR as previously described [22]. $\beta$-Actin (Mm01205647_g1) was used as the housekeeping gene. Data were obtained as cycle threshold $(\mathrm{Ct})$ values and were analyzed using the $\Delta \Delta \mathrm{Ct}$ method.

\section{Histological Analysis}

Adipocyte size was calculated on hematoxylin/eosin-stained (H\&E) sections $(7 \mu \mathrm{m})$ as follows: the area of interest was marked, and then cells were manually counted. The average cell size was calculated as the ratio of the marked area divided by the total count in that area. Liver steatosis was quantified on $\mathrm{H} \& \mathrm{E}$ sections $(4 \mu \mathrm{m})$ as unstained area per section area. Staining for F4/40 and ZO- 1 was performed as described previously [22].

\section{Statistical Analysis}

Data are shown as mean \pm SEM. A Kruskal-Wallis test, followed by Dunn's multiple comparisons test or a nonparametric 2-way ANOVA, was used when appropriate. Values of $p<0.05$ were considered statistically significant. Statistical analysis was performed using Prism 8 (GraphPad Software Inc., San Diego, CA, USA).

\section{Results}

\section{An HSUD, in Contrast to a WD or HFD, Induces Less Obesity, AT Inflammation, and Metabolic Abnormalities}

First, we confirmed that the WD indeed induces obesity. Mice fed with a WD had gained significantly more weight, had a larger total AT percentage, larger SC, GN, and MES AT weights, and larger adipocytes than mice fed with a standard-fat diet or a maintenance diet, even after correction for different caloric intakes (see online suppl. Fig. 1ae; for all online suppl. material, see www.karger. com/doi/10.1159/000516630). Furthermore, plasma cholesterol, hepatic steatosis, and the HOMA-IR index were highest in the WD group, indicating that a WD indeed induces obesity (online suppl. Fig. 1fh).

Next, the effects of HFD and HSUD on obesity development were studied. After 15 weeks of diet, mice on HSUD had a lower body weight and a lower total abdominal AT than mice on WD or HFD (Table 2; Fig. 1a, b). Furthermore, an HSUD resulted in lower SC, GN, and MES AT masses and larger adipocytes than a WD (Table 2). Scatter dot plots show that there is variability within each group, but that there are only a few nonresponders (online suppl. Fig. 2a-c). 
Obesity causes a lot of comorbidities, such as cardiovascular disease, type 2 diabetes, and NAFLD. Thus, our next aim was to investigate the development of these metabolic complications. An HSUD induced lower plasma cholesterol levels than a WD (Table 2). Additionally, the HOMA-IR index, a surrogate for insulin resistance, was lower in the HSUD group than in the WD group, whereas plasma adiponectin, which inversely correlates with insulin resistance, was higher in HSUD mice than in WD mice (Table 2). This decreased insulin resistance was confirmed with a glucose tolerance test (Fig. 1h). HSUD and HFD also induced less hepatic lipid accumulation than a WD (Fig. 1b, c). Next, we investigated the metabolic effects of the diets with indirect calorimetry. In this study, the SFD was used as control since a WD decreases ambulatory activity [23]. $\mathrm{CO}_{2}$ production, energy expenditure, and ambulatory activity were higher in the HSUD mice than in WD mice, but no differences were found between the HSUD and SFD, demonstrating a clear metabolic effect of the diets (Fig. 1i-k).

Intra-abdominal AT inflammation is a key feature of obesity and correlates with metabolic risk. In GN AT, mRNA levels of the pro-inflammatory markers MCP-1 and Saa3 were lower in the HSUD group than in the WD group (Fig. 1e). Saa3 expression also was lower in the HSUD group than in the HFD group (Fig. 1e). In MES AT, F4/80 and Saa3 mRNA levels were lower in the HSUD group than in the WD group (Fig. 1f). Furthermore, the number of crown-like structures (CLS), a hallmark of AT inflammation, was lower in the HSUD group than in the WD or HFD group (Fig. 1b, d).

Table 2. Effect of diet composition on organ weights and metabolic parameters

\begin{tabular}{|c|c|c|c|c|c|c|}
\hline & WD & HSUD & HSTD & HFRD & HCFD & HFD \\
\hline Body weight, g & $46 \pm 1$ & $36 \pm 1^{* *,{ }^{\circ}}$ & $32 \pm 1 * * * *$ & $38 \pm 1^{\$ \$}$ & $37 \pm 1^{*}$ & $45 \pm 3$ \\
\hline GN weight, mg & $2,626 \pm 136$ & $1,718 \pm 221^{*,^{\circ}}$ & $1,080 \pm 140^{* * * *}$ & $1,836 \pm 79^{\$}$ & $1,743 \pm 154$ & $2,701 \pm 294$ \\
\hline MES weight, mg & $1,117 \pm 81$ & $642 \pm 90^{*}$ & $525 \pm 64^{* * *}$ & $708 \pm 37$ & $726 \pm 68$ & $1,302 \pm 252$ \\
\hline SC weight, mg & $1,430 \pm 76$ & $855 \pm 100^{*}{ }^{\circ}$ & $575 \pm 66^{* * * *}$ & $995 \pm 33^{\$}$ & $1,006 \pm 88^{\$}$ & $1,775 \pm 305$ \\
\hline Liver weight, $\mathrm{mg}$ & $3,230 \pm 352$ & $2,229 \pm 177$ & $1,391 \pm 95^{* * * *}$ & $2,394 \pm 108^{\$ \$}$ & $2,538 \pm 168^{\$ \$}$ & $2,684 \pm 466$ \\
\hline GN adipocyte size, $\mu \mathrm{m}^{2}$ & $4,603 \pm 245$ & $3,131 \pm 266^{* *, \circ}$ & $2,637 \pm 222^{* * * *}$ & $3,374 \pm 274$ & $3,742 \pm 305$ & $4,568 \pm 501$ \\
\hline MES adipocyte size, $\mu \mathrm{m}^{2}$ & $3,067 \pm 201$ & $2,009 \pm 220^{* \circ}$ & $2,018 \pm 99^{* * * *}$ & $2,485 \pm 116$ & $2,338 \pm 143^{*}$ & $3,612 \pm 479$ \\
\hline SC adipocyte size, $\mu \mathrm{m}^{2}$ & $3,299 \pm 150$ & $2,458 \pm 246^{\circ}$ & $1,662 \pm 220^{*}$ & $2,124 \pm 212$ & $2,548 \pm 276$ & $3,788 \pm 307$ \\
\hline Cholesterol, mg/dL & $170 \pm 12$ & $95 \pm 9 * *$ & $103 \pm 9 * * *$ & $121 \pm 6^{*}$ & $119 \pm 10^{* *}$ & $142 \pm 21$ \\
\hline HOMA-IR index & $42 \pm 3$ & $15 \pm 2^{* *}$ & $4 \pm 2^{* * *}$ & $17 \pm 3$ & $14 \pm 5$ & $34 \pm 9$ \\
\hline Adiponectin, $\mu \mathrm{g} / \mathrm{mL}$ & $6 \pm 3$ & $6 \pm 2^{\circ}$ & $9 \pm 7^{*}$ & $7 \pm 6$ & $7 \pm 4$ & $5 \pm 3$ \\
\hline LPS levels, EU/mL & $11 \pm 0.6$ & $4 \pm 0.7^{* * *,{ }^{\circ}}$ & $5 \pm 0.4^{* *}$ & $5 \pm 0.5^{* *}$ & $4 \pm 0.4^{* *}$ & $8 \pm 0.5$ \\
\hline
\end{tabular}

$p$ values were calculated using the Kruskal-Wallis test followed by Dunn's multiple comparison's test, and results were considered significant when $p<0.05$. Data are presented as mean \pm SEM, and $n=10$ per group. GN, gonadal; MES, mesenteric; SC, subcutaneous; HOMA-IR, Homeostatic Model of Assessment-Insulin resistance; LPS, lipopolysaccharides. *Significantly different compared to WD. ${ }^{\circ}$ Significantly different compared to HFD. ${ }^{\$}$ Significantly different compared to HSTD. ${ }^{*}{ }^{\circ}, \$ p<0.05 .{ }^{* *} \$ \$ \$<0.01 .{ }^{* * *} p<0.001$. **** $p<0.0001$.

Fig. 1. The effect of HSUD, HFD, and WD on the development of obesity and obesity-associated metabolic complications. Quantification (a) of micro-CT images to visualize total body AT of mice kept on WD, HSUD, and HFD (b, top row) ( $n=2-4$ per group). AT is depicted in dark gray, indicated with red arrows. Quantification (c) of H\&E-stained liver sections (b, middle row) $(n=10$ per group). Images were taken at $\times 200$ (scale bar, $100 \mu \mathrm{m})$. Quantification (d) of F4/80-stained GN AT sections (b, bottom row). CLS are indicated with red arrows. Images were taken at $\times 200$ (scale bar, $100 \mu \mathrm{m}$ ). mRNA levels of F4/80, MCP-1, and Saa3 in GN (e) and MES (f) AT and of Occln, ZO-1, and Saa3 in colon tissue (g). Glu-

The Effect of Carbohydrates on Adipose

Tissue Inflammation cose tolerance test (h) of mice kept on HSUD or WD for 15 weeks ( $n=6$ per group). $\mathrm{CO}_{2}$ production (i), energy expenditure $(\mathbf{j})$, and ambulatory activity (k) of mice kept on WD, HSUD, or SFD for 14 weeks ( $n=6$ per group). $p$ values were calculated using the Kruskal-Wallis test followed by Dunn's multiple comparison's test (ag, i-k) or 2-way ANOVA (h), and results were considered significant when $p<0.05$. ${ }^{*} p<0.05 ;{ }^{* *} p<0.01 ;{ }^{* * *} p<0.001$; ${ }^{* * * *} p<$ 0.0001 . Data are presented as mean \pm SEM. WD, Western diet; HSUD, sucrose-rich diet; HFD, high-fat diet; SFD, standard-fat diet; AT, adipose tissue; GN, gonadal; MES, mesenteric; CLS, crown-like structures.

(For figure see next page.) 
a
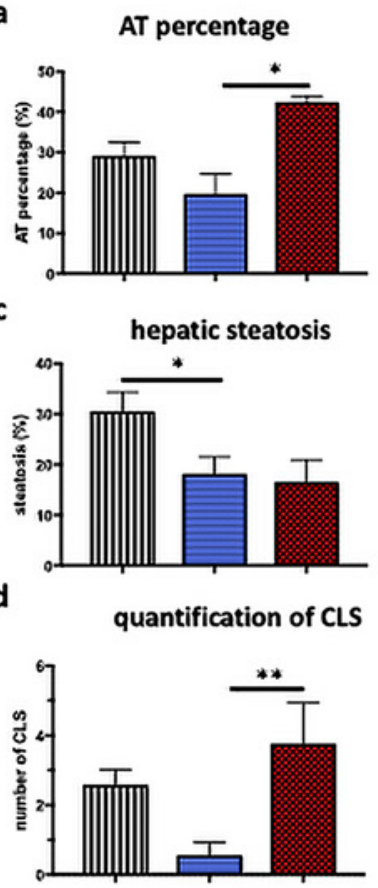

e

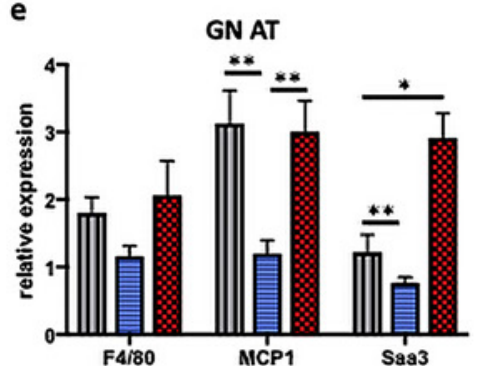

b
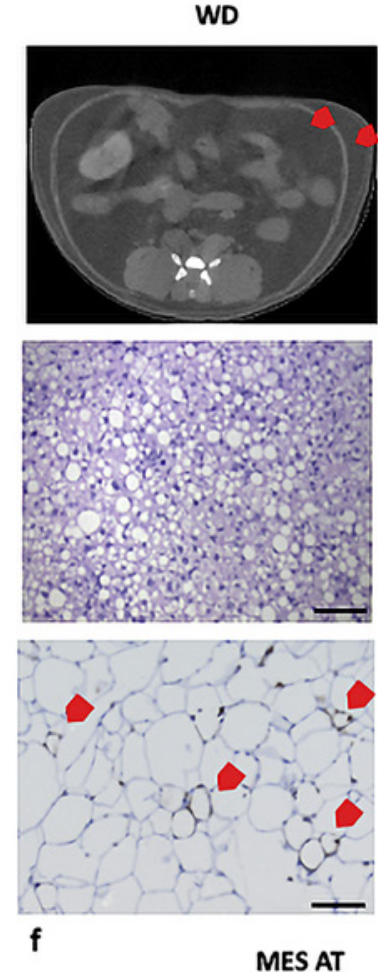
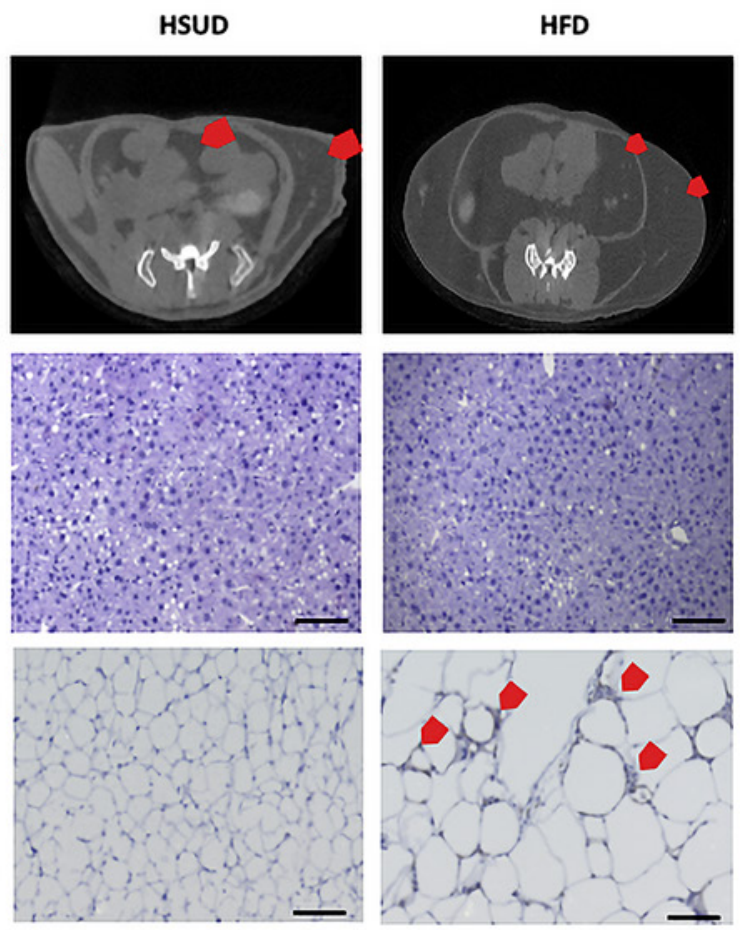

g
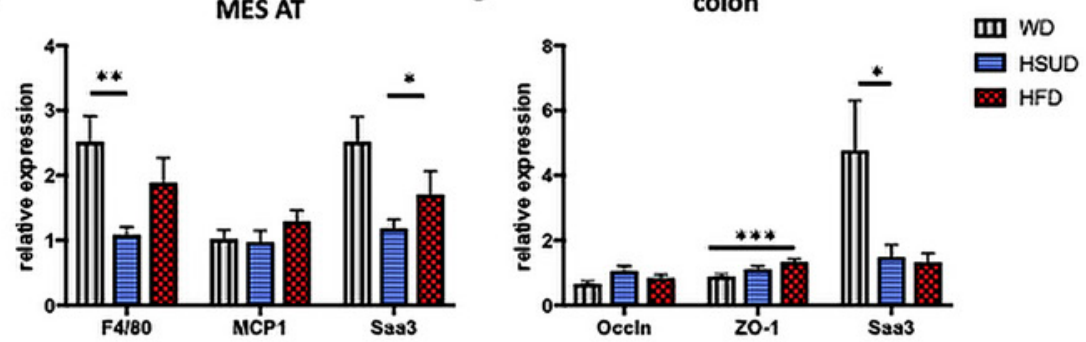

h
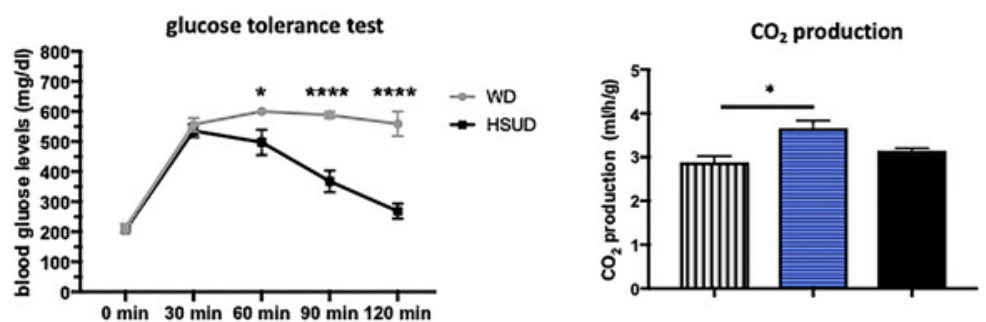

j
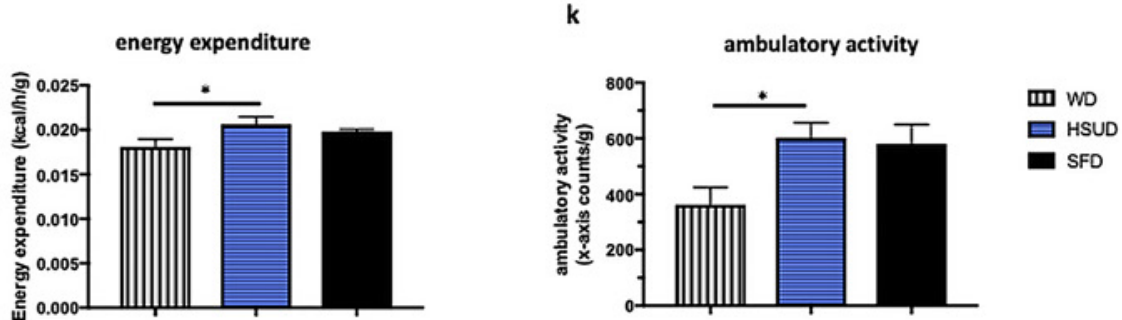


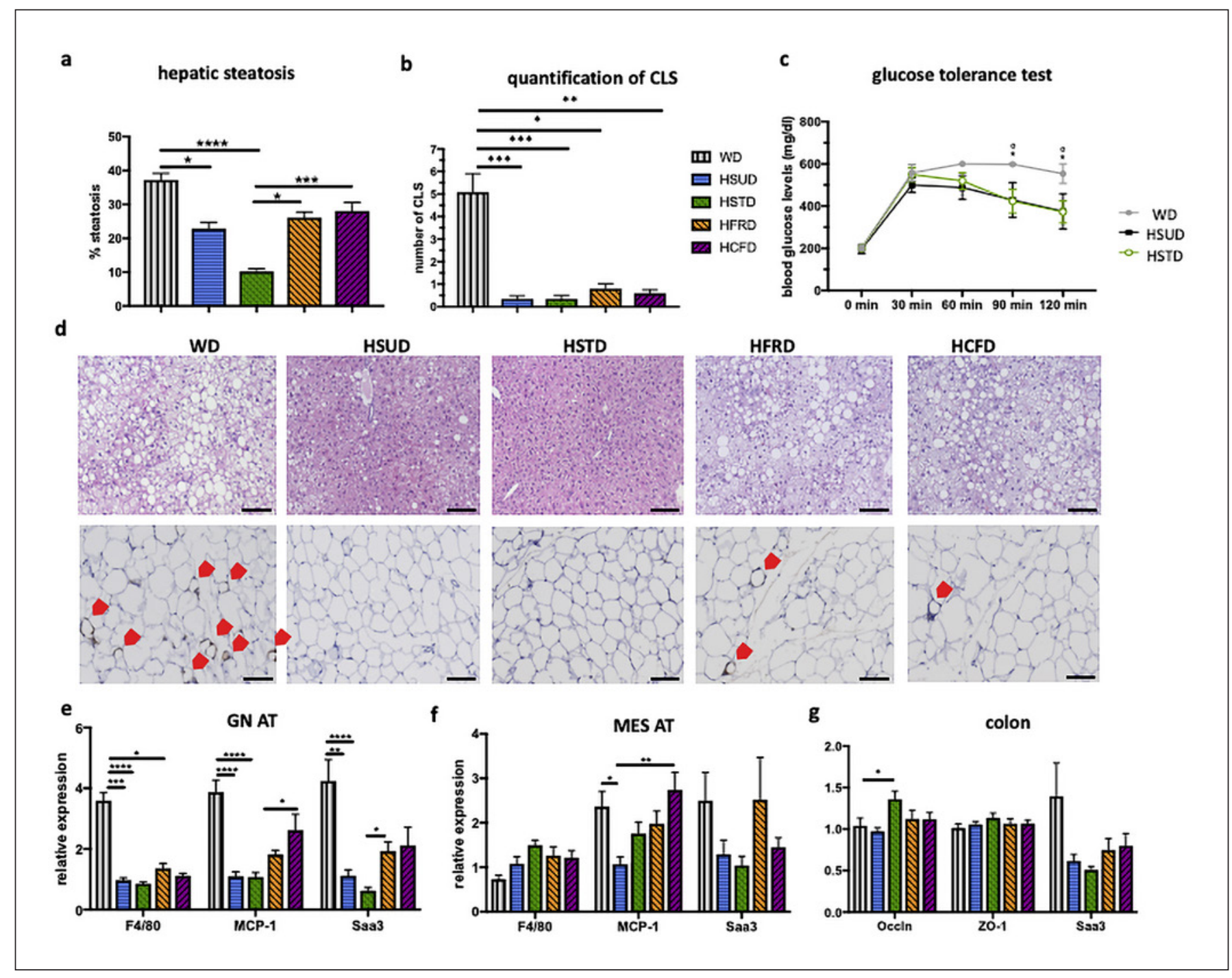

Fig. 2. The effect of different carbohydrate types on obesity, metabolism, AT inflammation, and intestinal integrity. Quantification (a) of H\&E-stained liver sections (d, top row) ( $n=10$ per group). Images were taken at $\times 200$ (scale bar, $100 \mu \mathrm{m})$. Quantification (b) of F4/80-stained GN AT sections (d, bottom row) ( $n=10$ per group). CLS are indicated with red arrows. Images were taken at $\times 200$ (scale bar, $100 \mu \mathrm{m})$. Glucose tolerance test (c) of mice kept on WD, HSUD, or HSTD for 15 weeks ( $n=10$ per group). mRNA levels of F4/80, MCP-1, and Saa3 in GN (e) and MES (f) AT and of Occln, ZO-1, and Saa3 in colon tissue ( $\mathbf{g})$ ( $n=10$ per group). $p$ values were calculated using the Kruskal-Wallis test followed by Dunn's multiple comparison's test (a, b, d-g) or 2-way ANOVA (c), and results were considered significant when $p<0.05 .{ }^{*} p<$ $0.05 ;{ }^{* *} p<0.01{ }^{* * *} p<0.001 ;^{* * * *} p<0.0001$. Data are presented as mean \pm SEM. WD, Western diet; HSUD, sucrose-rich diet; HSTD, high-starch diet; HFRD, high-fructose diet; HCFD, highfructose-sucrose diet; AT, adipose tissue; GN, gonadal; MES, mesenteric; CLS, crown-like structure.
It is evident that a WD decreases intestinal integrity, leading to an increased amount of LPS in the blood stream that aggravates the inflammatory state. But, how macronutrient composition exactly impacts intestinal integrity is not known yet. We found higher mRNA levels of the tight junction markers Occln and ZO-1 in colon tissue of mice on HSUD than of mice on WD (Fig. 1g). Further- more, colon Saa3 mRNA levels were lower in the HSUD group than the WD group (Fig. 1g). Next, we wanted to see if this better intestinal integrity also induced less LPS in the blood stream. Indeed, we found lower plasma LPS levels in the HSUD group than in the WD group (Table 2). Taken together, these data demonstrate that an HSUD induces less obesity and its complications such as 
Table 3. Effect of 8 weeks of caloric restriction and change of diet composition on organ weights and metabolic parameters of obese mice

\begin{tabular}{lccccc}
\hline & WD (control) & CR on HSUD & CR on HSTD & CR on WD & HSUD without CR \\
\hline GN weight, $\mathrm{mg}$ & $2,236 \pm 134$ & $804 \pm 100^{* * * *}$ & $888 \pm 94^{* * *}$ & $1,350 \pm 158$ & $1,534 \pm 134^{*}$ \\
MES weight, $\mathrm{mg}$ & $921 \pm 108$ & $271 \pm 40^{* * *}$ & $311 \pm 39^{* *}$ & $467 \pm 87$ & $450 \pm 71^{*}$ \\
SC weight, $\mathrm{mg}$ & $1,609 \pm 108$ & $567 \pm 49^{* * *}$ & $615 \pm 53^{* * *}$ & $797 \pm 105^{*}$ & $1,032 \pm 111^{* *}$ \\
Liver weight, mg & $4,105 \pm 441$ & $1,442 \pm 82^{* *}$ & $1,369 \pm 123^{* * *}$ & $1,358 \pm 87^{* * *}$ & $2,331 \pm 398^{*}$ \\
GN adipocyte size, $\mu \mathrm{m}^{2}$ & $5,657 \pm 409$ & $3,078 \pm 188^{* * *}$ & $2,934 \pm 249^{* * *}$ & $3,703 \pm 280$ & $4,381 \pm 126^{* *}$ \\
MES adipocyte size, $\mu \mathrm{m}^{2}$ & $2,850 \pm 194$ & $1,767 \pm 187^{*}$ & $1,538 \pm 148^{* *}$ & $2,024 \pm 198$ & $2,037 \pm 110^{*}$ \\
Cholesterol, $\mathrm{mg} / \mathrm{dL}$ & $216 \pm 23$ & $91 \pm 9^{* *}$ & $96 \pm 9^{* *}$ & $97 \pm 10^{* *}$ & $126 \pm 16^{*}$ \\
Adiponectin, $\mu \mathrm{g} / \mathrm{mL}$ & $6 \pm 0.5$ & $9 \pm 0.5^{* *}$ & $9 \pm 0.5^{* * *}$ & $8 \pm 0.3$ & $7 \pm 0.3^{*}$ \\
\hline
\end{tabular}

$p$ values were calculated using the Kruskal-Wallis test followed by Dunn's multiple comparison's test or Mann-Whitney U test (HSUD [no CR]), and results considered significant when $p<0.05$. Data are presented as mean \pm SEM; $n=10$ per group; $n=5$ in HSUD (no CR) group. CR, caloric restriction; GN, gonadal; MES, mesenteric; SC, subcutaneous; AT, adipose tissue. ${ }^{*} p<0.05$. ${ }^{* *} p<0.01$. ${ }^{* * *} p<0.001$. $* * * * p<0.0001$.

comorbidities, AT inflammation, and increased plasma LPS than a WD.

\section{Different Carbohydrates Exert Different Effects on}

Obesity Development and AT Inflammation

Since we observed beneficial effects of an HSUD, we next investigated the effect of different carbohydrate types. Mice on an HSUD or HSTD had lower body weights, smaller AT masses, and smaller adipocytes than mice on a WD (Table 2). Additionally, mice on HSTD also had lower AT weights than mice on HFRD (Table 2). Scatter dot plots show that there is variability within each group, but that there are only a few nonresponders (online suppl. Fig. 2df).

Next, we evaluated the effects of the different carbohydrates on the metabolic consequences of diet-induced obesity. Plasma cholesterol and the HOMA-IR index were lower in the HSUD and HSTD group than in the WD group (Table 2). Additionally, plasma adiponectin was lower in the WD group than in the HSTD group (Table 2). This lower insulin resistance in HSUD- and HSTDfed mice was confirmed with a glucose tolerance test (Fig. 2c). Hepatic steatosis was lower in the HSUD and HSTD group than in the WD group. Furthermore, there also was less steatosis in the HSTD group than in the HFRD and HCFD groups (Fig. 2a, d).

In accordance with the previous study, an HSUD induced lower F4/80, MCP-1, and Saa3 mRNA levels in GN AT than a WD (Fig. 2e). Furthermore, an HSTD also induced lower mRNA levels of these markers than a WD (Fig. 2e). In MES AT, a lower MCP-1 expression was found in the HSUD group than in the WD group (Fig. 2f).
The number of CLS in GN AT was also lower in all carbohydrate-rich groups than in the WD group (Fig. 2b, d). When analyzing the impact of our diets on the colon, we found a higher expression of Occln in the HSTD group as compared to the WD group (Fig. 2g). Additionally, plasma LPS levels were higher in the Western group than in all carbohydrate-rich groups (Table 2). Overall, these data clearly demonstrate that HSUD and HSTD induce less obesity and its consequences than a WD and that the type of carbohydrate is important in this prevention.

\section{A Carbohydrate-Rich Intervention Can Decrease Obesity and Its Complications}

Our data indicate that a carbohydrate-rich diet can prevent the development of obesity and its complications. Therefore, we next designed 2 carbohydrate-rich interventions to reduce the metabolic risk after mice were already obese. In the first intervention, obese mice were subjected to caloric restriction on a WD, an HSUD, or an HSTD for 8 weeks. All 3 caloric-restricted groups lost weight and had smaller AT masses and adipocytes, but differences were only significant if mice were switched to an HSUD or HSTD (Table 3; Fig. 3a, b). Scatter dot plots show that there is variability within each group, but that there are only a few nonresponders (online suppl. Fig. $2 \mathrm{~g}-\mathrm{i})$. Furthermore, caloric restriction on HSUD or HSTD induced less hepatic steatosis, lower plasma cholesterol, and higher plasma adiponectin levels than a WD without any restriction (Table 3 ). We found lower GN F4/80, MCP-1, and Saa3 mRNA levels as well as a smaller number of CLS in all caloric-restricted groups, regardless of diet composition (Fig. 3c, g). Moreover, in MES AT, 
a

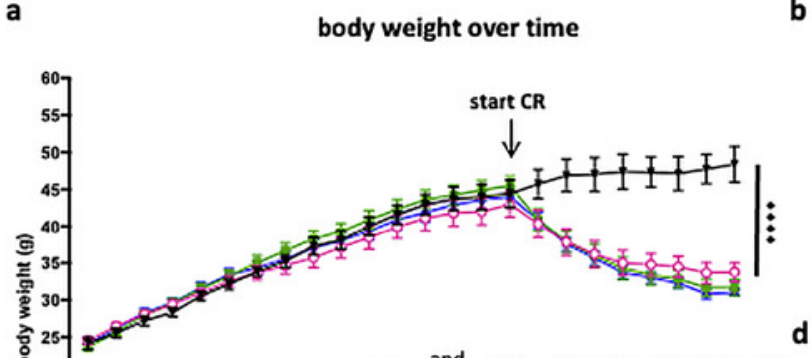

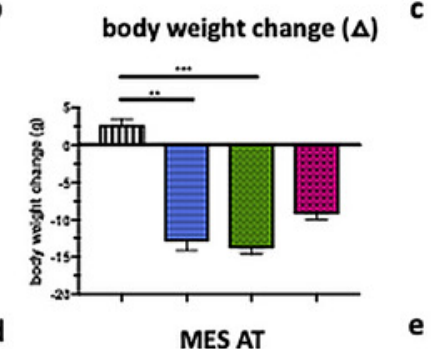

\section{c}

GN AT
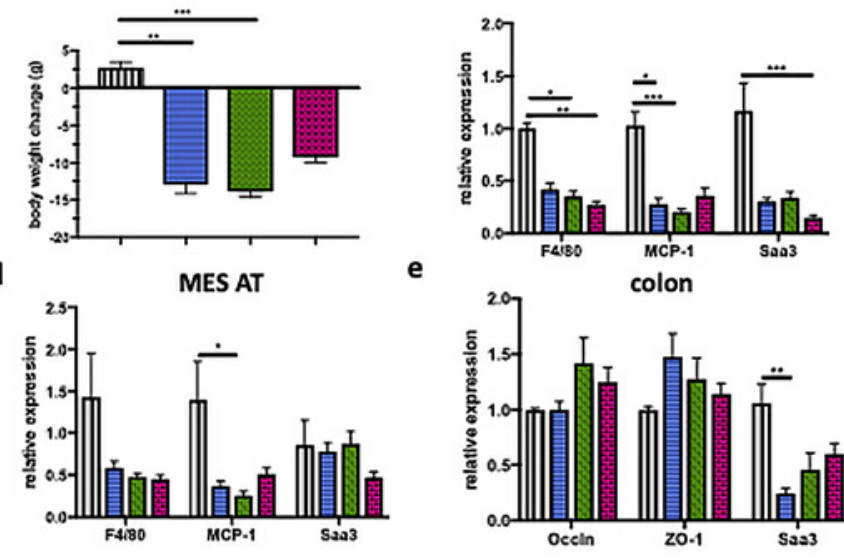

WD without CR (control)

CR on HSUD

CR on HSTD
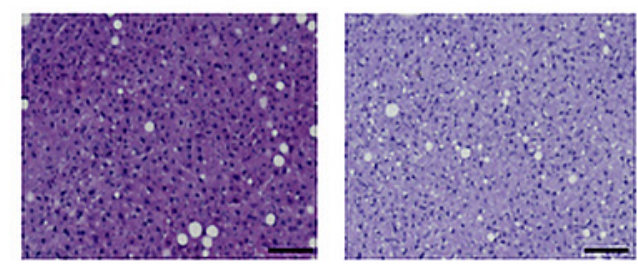

CR on WD

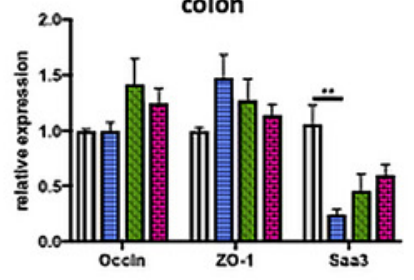

hepatic steatosis
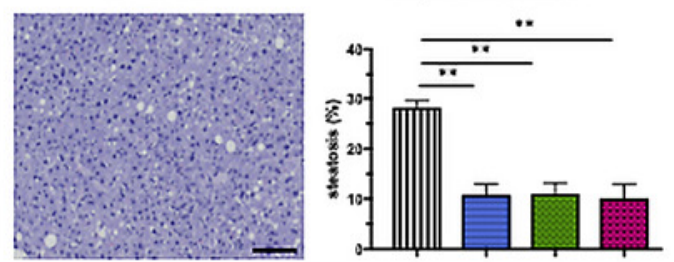

CR on western diet

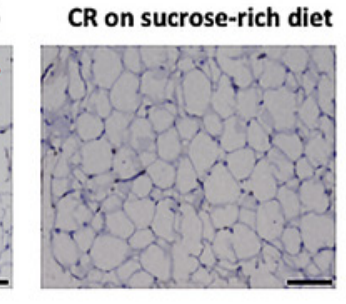

CR on high-starch diet
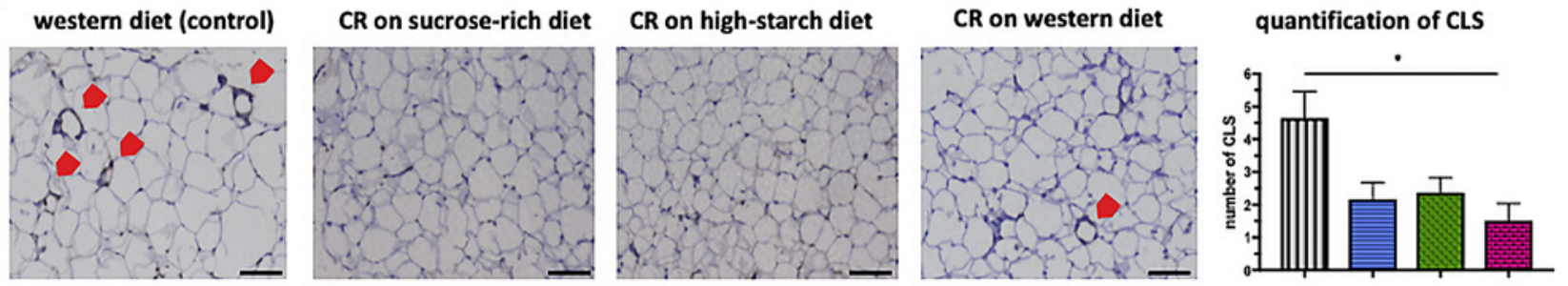

Fig. 3. The effect of caloric restriction on obesity, metabolism, AT inflammation, and intestinal integrity. The effect of 8 weeks of caloric restriction on body weight over time (a) and body weight change (b) in obese mice. mRNA levels of F4/80, MCP-1, and Saa3 in GN (c) and MES (d) AT and of Occln, ZO-1, and Saa3 in colon tissue (e) ( $n=10$ per group). Images (f) and quantification of $\mathrm{H} \& \mathrm{E}$-stained liver sections ( $n=10$ per group). Images were taken at $\times 200$ (scale bar, $100 \mu \mathrm{m})$. Images $(\mathbf{g})$ and quantification of CLS

MCP-1 mRNA levels were also lower in the caloric-restricted groups than in the controls, indicating that caloric restriction effectively reduced AT inflammation (Fig. 3d). No differences were found in colon Occln or ZO-1 mRNA levels, but Saa3 levels were lower in caloricrestricted mice than in controls (Fig. 3e). These data show that a caloric restriction reduces obesity and its complications and that effects are more pronounced with a caloric restriction on HSUD or HSTD.

The Effect of Carbohydrates on Adipose Tissue Inflammation in GN AT. Images were taken at $\times 200$ (scale bar, $100 \mu \mathrm{m}$ ), and red arrows point to CLS. $p$ values were calculated using the KruskalWallis test followed by Dunn's multiple comparison's test, and results were considered significant when $p<0.05 .{ }^{*} p<0.05 ;{ }^{* *} p<$ $0.01{ }^{* * *} p<0.001$. Data are presented as mean \pm SEM. WD, Western diet; HSUD, sucrose-rich diet; HSTD, high-starch diet; CR, caloric restriction; CLS, crown-like structures; GN, gonadal; MES, mesenteric; AT, adipose tissue.

For the second intervention, obese mice were switched to an HSUD without any caloric restriction. After 8 weeks, mice had lost significant weight, accompanied by smaller AT masses and adipocytes, even after correction for differences in caloric intake (Table 3; Fig. 4a, b). Scatter dot plots show that there is variability within each group, but that there are only a few nonresponders (online suppl. Fig. 2jl). Moreover, the switch to HSUD decreased hepatic steatosis, plasma cholesterol levels, and the HOMA- 
a

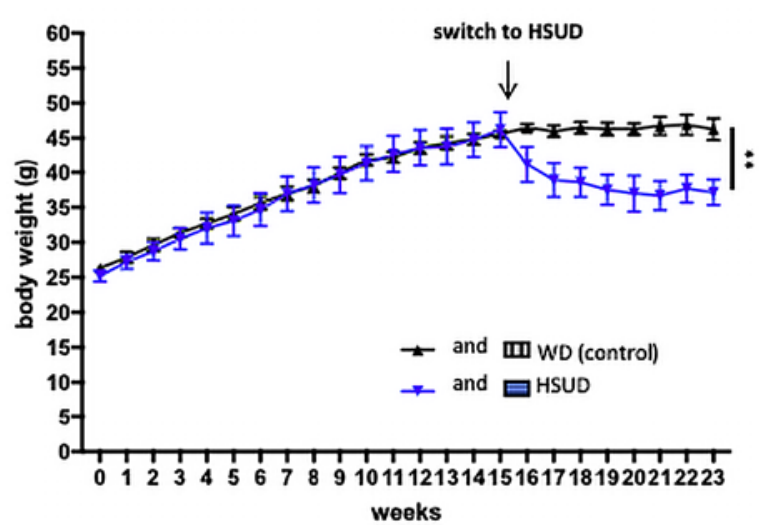

f
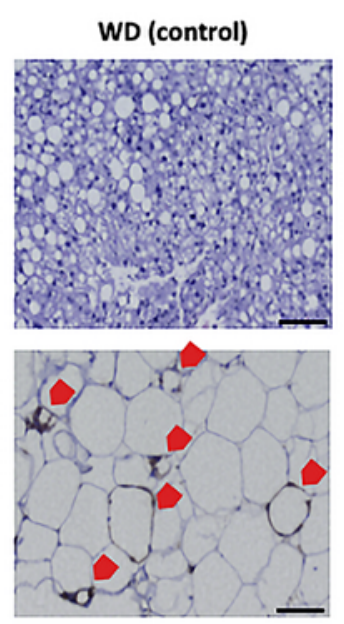

HSUD

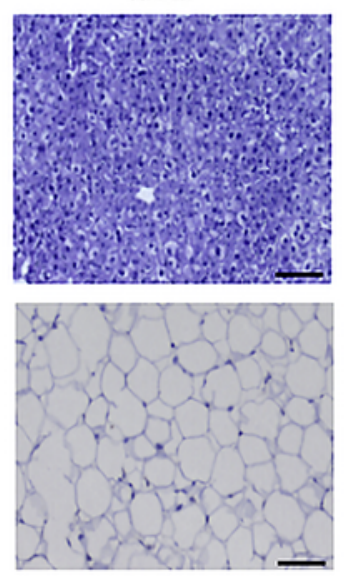

b

body weight change $(\Delta)$
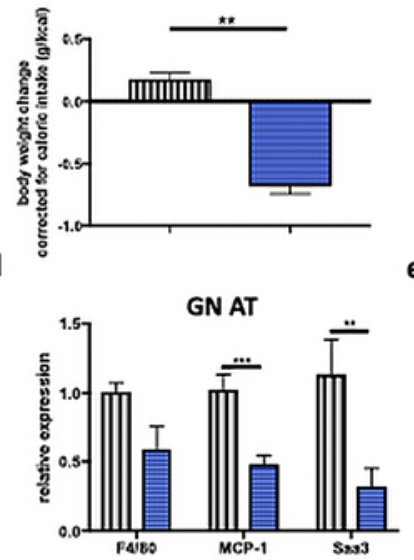

g

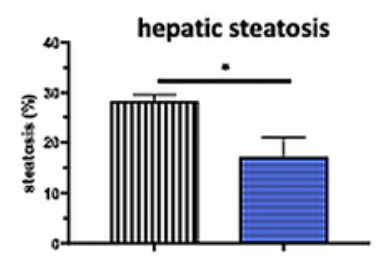

h

i quantification of CLS

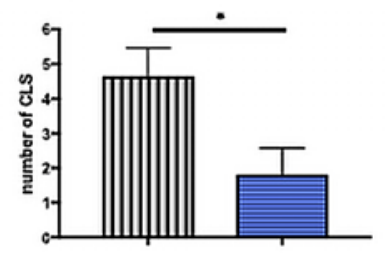

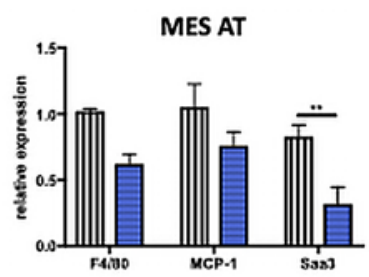
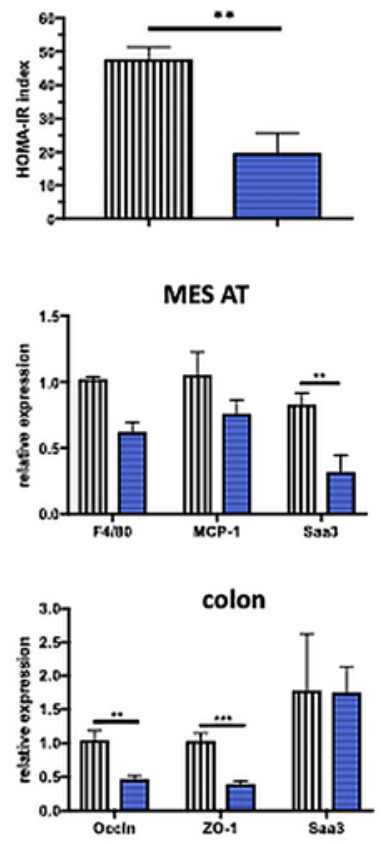

HOMA-IR index

j

plasma LPS levels

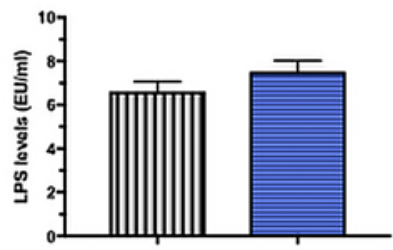

Fig. 4. The effect of diet composition switch on obesity, metabolism, AT inflammation, and intestinal integrity. The effect of 8 weeks of switch to an HSUD on body weight over time (a), body weight change (b), and HOMA-IR index (c) in obese mice $(n=5$ per group). mRNA levels of F4/80, MCP-1, and Saa3 in GN (d) and MES (e) AT and of Occln, ZO-1, and Saa3 in colon tissue (h) $(n=$ 5 per group). Images (f, top row) and quantification (g) of H\&Estained liver sections ( $n=5$ per group). Images were taken at $\times 200$ (scale bar, $100 \mu \mathrm{m}$ ). Images (f, bottom row) and quantification (i) of CLS in GN AT. Images were taken at $\times 200$ (scale bar, $100 \mu \mathrm{m}$ ), and red arrows point to CLS. Plasma LPS levels (j). $p$ values were calculated with 2-way ANOVA (a) or Mann-Whitney U test (b-i), and results were considered significant when $p<0.05{ }^{*} p<0.05$; ${ }^{* *} p<0.01 ;{ }^{* * *} p<0.001$. Data are presented as mean \pm SEM. WD, Western diet; HSUD, sucrose-rich diet; CLS, crown-like structures; GN, gonadal; MES, mesenteric; AT, adipose tissue; LPS, lipopolysaccharides.
IR index and increased plasma adiponectin levels (Table 3; Fig. 4c, f, g). In both GN and MES AT, mRNA levels of F4/80, MCP-1, and Saa3 were lower in the HSUD group than in the WD group (Fig. 4d, e). Furthermore, the number of CLS was also lower after the switch to the HSUD (Fig. 4f, i). No differences were found in colon Occln or ZO-1 levels or plasma LPS levels (Fig. 4h, j). These results demonstrate that a switch to an HSUD is sufficient to reduce obesity and obesity-induced complications, even without caloric restriction.

We correlated the body weight at the end of all of the studies to investigate whether the lower body weights of the mice kept on the different carbohydrate-rich diets and of the mice that underwent the interventions correlated with lower GN, MES, and SC AT weight (online suppl. Fig. 3ac). The $R$ value was very close to $1(0.81-0.90)$ in- 
dicating a strong positive correlation between body weight and AT depot weights. Additionally, we also found a strong correlation between body weight and plasma cholesterol, adiponectin, insulin, and the HOMA-IR index, indicating that body weight correlates with metabolic risk in our studies (online suppl. Fig. 3dg).

\section{Discussion}

In this study, we demonstrated that in a murine model of diet-induced obesity, a carbohydrate-rich diet is able to prevent and treat obesity and its metabolic complications. Before diving into the main research question "What is the effect of macronutrient composition on obesity and its metabolic complications?," we first confirmed that our WD indeed effectively induced obesity. In this experiment, 2 control diets were included: the SFD and the MD. The SFD was lower in carbohydrate and fat content than the WD, but this meant an increased protein level since there are only 3 macronutrients that make up diet composition. The MD was included because it is the most frequently used control diet in the literature. However, because of its high carbohydrate content of $67.5 \%$, it was not a suitable control diet for our research question. Therefore, we decided to use the WD as positive control in the study because it induces obesity and it has the same protein content as the other diets, excluding protein content as a confounding factor.

We showed that HSUD and HSTD induced less obesity and its complications than a WD. Our data are in accordance with a study by $\mathrm{Hu}$ et al. [12] that demonstrated that fat, but not carbohydrates, causes adiposity in mice. Human studies have reported conflicting results. In the PURE study, a higher carbohydrate intake, but not a higher fat intake, was associated with an increased risk of total mortality [24]. In contrast, a study by Smith et al. [25] revealed no association between carbohydrate intake and energy balance. Furthermore, several studies showed no association between carbohydrate intake and overweight or obesity $[6,10,12,26,27]$. The discrepancy between murine and human data can at least partly be explained by a lack of adherence to diet in human studies, highlighting the necessity of murine models for dietary studies to develop evidence-based dietary guidelines [28].

In our study, an HFRD did not induce obesity, but we did observe increased hepatic steatosis. This is in line with the existing literature, since the link between a high-fructose intake and hepatic damage has been well documented [29]. Fructose metabolism occurs almost exclusively

The Effect of Carbohydrates on Adipose

Tissue Inflammation via the liver. Therefore, its effects on blood glucose levels are rather limited [30]. But, because it is metabolized differently, it does promote ectopic lipid accumulation upon excess intake, especially in the liver and skeletal muscle [30]. Therefore, it is evident that a high fructose intake promotes obesity and its associated comorbidities, especially NAFLD $[31,32]$.

We demonstrated that an HSTD induces a better health status in mice than a WD. This is contradictory to what is known in humans. Both preclinical and clinical studies have demonstrated that a high intake of rapidly digestible starches, such as boiled potatoes, white bread, and refined grains, positively correlate with obesity $[2,33$, 34]. A possible explanation for this discrepancy is the diet we used. A WD is characterized by not only a high carbohydrate intake, mostly from sucrose, fructose, and rapidly digestible starchy foods, but also by a high dietary fat intake. In our study, fat intake was kept very low (10\%). Moreover, our diets were designed to contain all necessary micronutrients, and this is often not the case in a WD [2]. It should be noted that in our study, the carbohydrates were included in the diet. The effects probably would be different if a high-carbohydrate intake would be achieved via the drinking water. The detrimental effects of sugar-sweetened beverages have been well established, whereas the impact of carbohydrates, especially simple carbohydrates such as sucrose and fructose, ingested via foods is still relatively unclear [35].

Mice fed with an HFD did develop obesity and some obesity-associated complications, although the severity of these complications was lower than of those induced by a WD. We specifically designed our HFD to contain as much fat as possible, without inducing ketogenesis, since it has been demonstrated that ketogenic diets can induce weight loss [36, 37]. Furthermore, because the benefits of a diet high in unsaturated fats have been well established and a WD contains a large proportion of saturated fats, we chose to focus on the effects of these saturated fats.

In contrast to our findings, Kang et al. [38] showed that sucrose induces more AT inflammation than an HFD without sucrose. Additionally, Oliveira et al. [39] reported that in mice, a high-sucrose diet did not lead to obesity, but did increase serum MCP-1 expression, indicating inflammation. However, in these studies, sucrose content was only $32-37 \%$ and fat content was higher, resembling more a WD than a sucrose-rich diet. Our HSUD was much higher in sucrose content (75\%).

In accordance with the existing literature, we confirmed that a WD decreased intestinal integrity and that 
a carbohydrate-rich diet ameliorates intestinal integrity [18]. Furthermore, Saa3 expression was lower in colon tissue of WD-fed mice than in that of mice fed with a carbohydrate-rich diet. Felin et al. [40] and Reigstadt and Bäckhed [41] demonstrated that the pro-inflammatory Saa3 is not only expressed in AT but also in the colon, where it can bind bacteria. These data indicate a role for Saa3 in intestinal permeability and a possibility for a manipulation of Saa3 levels through diet composition.

In the third part of the study, we aimed to reduce the WD-induced metabolic risk via caloric restriction and/or change of diet composition. In accordance with the existing literature, caloric restriction induced weight loss and a decrease in metabolic complications as well as AT inflammation in obese mice, regardless of macronutrient composition [42, 43]. A switch to an HSUD after obesity induction induced significant weight loss, ameliorated the metabolic health status, and reduced AT inflammation even without any caloric restriction. These results are in line with the existing literature, where a switch to a high-carbohydrate diet decreased obesity [18, 44, 45]. Furthermore, Johnstone et al. [46] showed that weight loss can be maintained with a diet higher in carbohydrates. Strikingly, neither a caloric restriction nor a switch to an HSUD markedly improved intestinal integrity. A switch to an HSUD also did not decrease metabolic endotoxemia, suggesting that the beneficial effects of the dietary intervention were exerted without an impact on the intestines. A recent study by Fragiadakis et al. [47] demonstrated that the gut microbiome could be altered by changing the diet composition on the short term, but on the longer term, it restored itself to the initial state. Additionally, Johnstone et al. [46] showed that even though different diet compositions influence the gut microbiome, all diets had similar efficacy in maintaining weight loss and improving health. Since the gut microbiome influences intestinal integrity, the resilience of gut microbiota composition might be a reason why we did not detect any differences in intestinal integrity or metabolic endotoxemia. But, to confirm or refute this hypothesis, additional studies with a focus on the gut microbiome and intestines would be necessary. In conclusion, this study highlights the importance of macronutrient composition in the battle against obesity and provides more insight into the effects of carbohydrates on the development of obesity and obesity-associated comorbidities as well as possibilities for carbohydrates in the treatment of obesity.

\section{Acknowledgments}

We would like to thank Dr. Bianca Hemmeryckx, Liesbeth Frederix, Max Gooijen, and Tatevik Suprikyan very much for all their help with the experiments.

\section{Statement of Ethics}

All animal procedures were approved by the Ethics Committee of the KU Leuven (P031/2017 and P106/2018) and performed in accordance with the NIH Guide for the Care and Use of Laboratory Animals (1996).

\section{Conflict of Interest Statement}

All authors declare that they have no conflicts of interest.

\section{Funding Sources}

This work was supported by "Programmafinanciering KU Leuven" under Grant (PF/10/014) and Onderzoeksraad KU Leuven (PF/10/014).

\section{Author Contributions}

Ellen Vercalsteren, Ilse Scroyen, and Roger Lijnen designed the experiments. Ellen Vercalsteren and Christine Vranckx performed the experiments. Bart Van der Schueren provided equipment, and Katrien Corbeels performed the indirect calorimetry. Greetje Vande Velde provided equipment and algorithms necessary for CT imaging and analysis. Ellen Vercalsteren performed statistical analysis. Ellen Vercalsteren and Ilse Scroyen wrote the manuscript. All authors reviewed, revised, and approved the manuscript.

\section{Data Availability Statement}

The data that support the findings of this study are available from the corresponding author (Ilse Scroyen), upon reasonable request.

\footnotetext{
References

1 Semenkovich CF. Insulin resistance and atherosclerosis. J Clin Invest. 2006;116(7):1813-22.

2 Mozaffarian D. Dietary and policy priorities for cardiovascular disease, and obesity: a comprehensice review. Circulation. 2017;133: 187-225.

3 Hotamisligil GS. Inflammation and metabolic disorders. Nature. 2006;444(7121):860-7.

4 Capel F, Viguerie N, Vega N, Dejean S, Arner P, Klimcakova E, et al. Contribution of energy restriction and macronutrient composition to changes in adipose tissue gene expression during dietary weight-loss programs in obese women. J Clin Endocrinol Metab. 2008;93(11):4315-22.
}

Vercalsteren/Vranckx/Corbeels/Van der Schueren/Velde/Lijnen/Scroyen 
5 Abete I, Astrup A, Matrinez J, Thorsdottir I, Zulet M. Obesity and the metbolic syndrome: role of different dietary macronutrient distribution patterns and specific nutritional components on weight loss and maintenance. Nutr Rev. 2010;68:214-31.

6 Pinho Lde, Andrade O, Paral A, Batista A, Filho M, John D, et al. Diet composition modulates expression of sirtuins and renin-angiotensin system components in adipose tissue. Obesity. 2013;21:1830-5.

7 Fortino MA, Lombardo YB, Chicco A. The reduction of dietary sucrose improves dyslipidemia, adiposity, and insulin secretion in an insulin-resistant rat model. Nutrition. 2007; 23(6):489-97.

8 Kuhnle GGC, Tasevska N, Lentjes MAH, Grif JL, Sims MA, Richardson L, et al. Association between sucrose intake and risk of overweight and obesity in a prospective sub-cohort of the European Prospective Investigation into Cancer in Norfolk ( EPIC-Norfolk). Public Health Nutr. 2015;18:2815-24.

9 WHO. Guideline: sugars intake for adults and children. 2015. Available from: https:// www.who.int/nutrition/publications/guideli.

10 Rippe JM, Angelopoulos TJ. Added sugars and risk factors for obesity, diabetes and heart disease. Int J Obes. 2016;40(S1):S22-7.

11 Sartorius K, Sartorius B, Madiba TE, Stefan C. Does high-carbohydrate intake lead to increased risk of obesity? A systematic review and meta-analysis. BMJ Open. 2018;8: e018449.

12 Hu S, Wang L, Yang D, Li L, Togo J, Wu Y, et al. Dietary fat, but not protein or carbohydrate, regulates energy intake and causes adiposity in mice. Cell Metab. 2018;28(3):41531.e4.

13 Vajro P, Paolella G, Fasano A. Microbiota and gut - liver axis: their influences on obesity and obesity-related liver disease. J Pediatr Gastroenterol Nutr. 2013;56:461-8.

14 Geurts L, Neyrinck AM, Delzenne NM, Knauf C, Cani PD. Gut microbiota controls adipose tissue expansion, gut barrier and glucose metabolism: novel insights into molecular targets and interventions using prebiotics. Benef Microbes. 2014;5(1):3-17.

15 Cani PD, Amar J, Iglesias MA, Poggi M, Knauf C, Bastelica D, et al. Metabolic endotoxemia initiates obesity and insulin resistance. Diabetes. 2007;56(7):1761-72.

16 Paula A, Moreira B, Fiche T, Texeira S, Ferreira $A B$, Gerais $M$. Influence of a high-fat diet on gut microbiota, intestinal permeability and metabolic endotoxaemia. Br J Nutr. 2012; 108:801-9.

17 Fava F, Gitau R, Griffin BA, Gibson GR, Tuohy KM, Lovegrove JA. The type and quantity of dietary fat and carbohydrate alter faecal microbiome and short-chain fatty acid excretion in a metabolic syndrome 'at-risk' population. Int J Obes. 2012;37(2):216-23.

18 Lopez-legarrea P, Fuller NR, Zulet MA, Martinez JA, Caterson ID. The influence of mediterranean, carbohydrate and high protein di- ets on gut microbiota composition in the treatment of obesity and associated inflammatory state. Asia Pac J Clin Nutr. 2014;23(3): $360-8$.

19 Yang L, Guo G, Niu X, Liu J. Dendritic cellbased immunotherapy treatment for glioblastoma multiforme. Biomed Res Int. 2015;2015: 717530.

20 Canfora EE, van der Beek CM, Hermes GDA, Goossens GH, Jocken JWE, Holst JJ, et al. Supplementation of diet with galacto-oligosaccharides increases bifidobacteria, but not insulin sensitivity, in obese prediabetic individuals. Gastroenterology. 2017;153(1):8797.e3.

21 Ferrannini E. The theoretical bases of indirect calorimetry: a review. Metabolism. 1988; 37(3):287-301.

22 Geys L, Roose E, Vanhoorelbeke K, Bedossa P, Scroyen I, Lijnen HR. Role of ADAMTS13 in diet-induced liver steatosis. Mol Med Rep. 2017;16(2):1451-8.

23 Hiramatsu L, Kay JC, Thompson Z, Singleton JM, Claghorn GC, Albuquerque RL, et al. Maternal exposure to Western diet affects adult body composition and voluntary wheel running in a genotype-specific manner in mice. Physiol Behav. 2017;179:235-45.

24 Dehghan M, Mente A, Zhang X, Swaminathan S, Li W, Mohan V, et al. Associations of fats and carbohydrate intake with cardiovascular disease and mortality in 18 countries from five continents ( PURE ): a prospective cohort study. Lancet. 2017;390:2050-62.

25 Smith HA, Gonzalez JT, Thompson D, Betts JA. Dietary carbohydrates, components of energy balance, and associated health outcomes. Nutr Rev. 2017;75(10):783-97.

26 Song W, Wang Y, Chung C, Song B, Lee W, Chun O. Is obesity development associated with dietary sugar intake in the U.S.? Nutrition. 2012;28:1137-41.

27 Farajian P, Risvas G, Panagiotakos DB, Zampelas A. Food sources of free sugars in children's diet and identification of lifestyle patterns associated with free sugars intake: the GRECO (Greek Childhood Obesity) study. Public Health Nutr. 2015;19:2326-35.

28 Speakman JR. Use of high-fat diets to study rodent obesity as a model of human obesity. Int J Obes. 2019;43(8):1491-2.

29 Tappy L. Fructose-containing caloric sweeteners as a cause of obesity and metabolic disorders. J Exper Biol. 2018;121:jeb164202.

30 Tappy L, Lê KA. Metabolic effects of fructose and the worldwide increase in obesity. Physiol Rev. 2010;90(1):23-46.

31 Tetri LH, Basaranoglu M, Brunt EM, Yerian LM, Neuschwander-tetri BA. Severe NAFLD with hepatic necroinflammatory changes in mice fed trans fats and a high-fructose corn syrup equivalent. Am J Physiol Gastrointest Liver Physiol. 2008;295:987-95.

32 Basaranoglu M, Basaranoglu G, Sabuncu T, Sentürk H. Fructose as a key player in the development of fatty liver disease. World J Gastroenterol. 2013;19(8):1166-72.
33 Hasek LY, Phillips RJ, Zhang G, Kinzig KP, Kim CY, Powley TL, et al. Dietary slowly digestible starch triggers the gut-brain axis in obese rats with accompanied reduced food intake. Mol Nutr Food Res. 2018;62(5).

34 Achour L, Flourié B, Briet F, Franchisseur C, Bornet F, Champ M, et al. Metabolic effects of digestible and partially indigestible corn starch: a study in the absorptive and postabsorptive periods in healthy. Am Soc Clin Nutr. 1997;66:1151-9.

35 Malik VS, Hu FB. Sugar-sweetened beverages and cardiometabolic health: an update of the evidence. Nutrients. 2019;11(8):1840.

36 Roberts MN, Wallace MA, Tomilov AA, Zhou Z, Marcotte GR, Tran D, et al. A ketogenic diet extends longevity and healthspan in adult mice. Cell Metab. 2017;26(3):539-46.

37 Gupta L, Khandelwal D, Kalra S, Gupta P, Dutta D, Aggarwal S. Ketogenic diet in endocrine disorders: current perspectives. J Postgrad Med. 2017 OctDec;63(4):242-51.

38 Kang I, Espín JC, Carr TP, Tomás-Barberán FA, Chung S. Raspberry seed flour attenuates high-sucrose diet-mediated hepatic stress and adipose tissue inflammation. J Nutr Biochem. 2016;32:64-72

39 Oliveira LSC, Santos DA, Barbosa-da-Silva S, Mandarim-de-Lacerda CA, Aguila MB. The inflammatory profile and liver damage of a sucrose-rich diet in mice. J Nutr Biochem. 2014;25(2):193-200.

40 Felin J, Ba F, Reigstad CS. Regulation of serum amyloid A3 ( SAA3 ) in mouse colonic epithelium and adipose tissue by the intestinal $\mathrm{mi}$ crobiota. PLoS One. 2009;4:e35842.

41 Reigstad CS, Bäckhed F. Microbial regulation of SAA3 expression in mouse colon and adipose tissue. Gut Microbes. 2010;1(1):55-7.

42 Thom G, Lean M. Is there an optimal diet for weight management and metabolic health? Gastroenterology. 2017;152(7):1739-51.

43 Muhammad HFL, Vink RG, Roumans NJT, Arkenbosch LAJ, Mariman EC, Van Baak MA. Dietary intake afterweight loss and the risk of weight regain: macronutrient composition and inflammatory properties of the diet. Nutrients. 2017;9:1205

44 Santos-Marcos JA, Perez-Jimenez F, Camargo $\mathrm{A}$. The role of diet and intestinal microbiota in the development of metabolic syndrome. J Nutr Biochem. 2019;70:1-27.

45 Griffin C, Hutch CR, Abrishami S, Stelmak D, Eter L, Li Z, et al. Inflammatory responses to dietary and surgical weight loss in male and female mice. Biol Sex Differ. 2019;10(1):16.

46 Johnstone AM, Kelly J, Ryan S, Romero-Gonzalez R, McKinnon H, Fyfe C, et al. Nondigestible carbohydrates affect metabolic health and gut microbiota in overweight adults after weight loss. J Nutr. 2020;150(7):1859-70.

47 Fragiadakis GK, Wastyk HC, Robinson JL, Sonnenburg ED, Sonnenburg JL, Gardner CD. Long-term dietary intervention reveals resilience of the gut microbiota despite changes in diet and weight. Am J Clin Nutr. 2020;111(6):1127-36. 